\author{
M. Budzik ${ }^{1,2}$, J. Jumel ${ }^{1}$, M.E.R. Shanahan ${ }^{1}$ \\ ${ }^{1}$ Université Bordeaux 1, Laboratoire de Mécanique Physique LMP-UMR CNRS 5469, 351 \\ Cours de la Libération, 33405 TALENCE Cedex, France \\ 2 Technical University of Gdansk, Faculty of Mechanical Engineering, Dept. of Material \\ Science and Engineering, Narutowicza 11/12, 80-233 Gdansk, Poland
}

\title{
FRACTURE ENERGY OF BONDED JOINTS WITH 2D ELASTIC ADHESIVE LAYER
}

\begin{abstract}
When bonded joint is subjected to mode I fracture loading, the adhesive joints analytical solutions treats the adhesive layer, usually, as not existing or 1D Hook elastic layer. In the case of 1D elastic layer, represented as Hooks spring element, is acting, only, in direction contrary to the applied load. Basing on the information yielded from sensitive laser profilometry technique, where deflections of bonded part of the joint were measured, within this contribution, 2D Finite Element Method model is introduced. The FEM allows adhesive layer to be simulated as two perpendicular-acting Hook's springs, thus in-plane shear compliance is enabled. Subsequently, appropriate analysis were carried out. Results, in terms of plate deflection, were compared with laser profilometry technique and common analytical solutions. It is concluded that linear 1D model is not sufficient for the asymmetric bonded joint configuration since the adhesive resists actively also in the in-plane shearing direction. Omitting shearing compliance effect can lead to valuable misinterpretation of the fracture energy, up to $20 \%$ in cases studied, and thus, cannot be ignored. Finally, power law based, correction factors are given promising fast and reliable data correction.
\end{abstract}

Keywords: bonded joints, energy release rate, finite element analysis, $2 D$ adhesive model.

\section{INTRODUCTION}

Adhesive bonding technique is well established joining method of secondary [e.g. 1-3] and primary structures $[$ e.g. 4, 5]. This is mainly due to attractive weight-to-mechanical properties ratio effecting in more efficient structures. The development in the adhesive science and technique is very complex, from e.g. adhesive formulation [6], surface preparation procedures [7], mechanical testing [8] and numerical modelling [e.g. 9, 10]. In the case of adhesive joint mechanics progress brings new knowledge about general mechanical behaviour of the bonded joints resulting in more complex and sophisticated models describing joint behaviour. In the fracture mechanics, when opening the most critical and common, mode I cleavage load is applied, the traditional experimental procedures use the solutions based on Euler-Bernoulli beam theory [e.g. 11, 12] and corrected beam theory [13]. These models do not take adhesive layer into account which is specifically required when compliant adhesives are used. Compliance effect can be introduced by treating adhesive layer as a Winkler elastic foundation $[14,15]$. Numerical and experimental observations proves that also other effects affecting fracture could be present when the joint is submitted to rather simple 1D loading 
$[16,17]$. The focus is more on sophisticated codes, that on possibility to prove and test the results obtained. From experimental and more industrial point of view, some fast and easy solutions are required. In the recent contribution finite element method is implemented and compared with two common analytical solutions of the beams used to simulate adhesive joints. This are applied to the asymmetric adhesive joints (the most common) loaded in mode I (cleavage) fracture mechanics direction. Thus, within this contribution, adhesive layer is treated as: not existing - classical Euler-Bernoulli model, 1D arrangement of Hook's spring elements - for Winkler model and finally as 2D elastic finite elements. Results of such made analysis, in terms of bonded plates deflections, are compared with newly applied laser profilometry technique. It was found that $1 \mathrm{D}$ analytical solution, when the beams deflection are accounted could be treated as satisfactory. However, in terms of fracture energy, the FEM and both analytical solutions gave different results leading to possible results misinterpretation. That was related to the possible 2D compliance of the adhesive when submitted to, theoretically, 1D loading. Finally, using power law fitting, correction factors of fracture energy are proposed. Factors which allows fast and reliable treatment of experimental results, where basic treatment must still be held within classical analytical solutions.

\section{EXPERIMENTAL}

\section{Materials and preparation}

An asymmetric wedge configuration was used (Fig.1a.) in which a flexible, aluminium alloy plate (Dural or Avional AA 2024 - T3) of thickness, $h=1.5 \mathrm{~mm}$, was bonded to a rigid plate (Hydronalium AA 5754) of thickness $H=6 \mathrm{~mm}$. Young's modulus of the thinner plate, $E$, obtained by 3 -point bending, was evaluated at $68 \pm 5 \mathrm{GPa}$. Poisson's ratio, $v$, obtained by ultra-sound TTM (through transmission method), was 0.32 . Relative flexural rigidity, given by the ratio of the cubes of thickness multiplied by the appropriate Young's modulus (here the same), leads to a value of $c a$. 64 . The flexible adherend (length, $l=120 \mathrm{~mm}$ ) was bonded to the rigid member along a length, $l_{\mathrm{adh}}=100 \mathrm{~mm}$. The latter was of length, $L=180 \mathrm{~mm}$. Initial effective crack length, $a$, of $c a$. $17.5 \mathrm{~mm}$ was achieved. The entire system was of width, $b=$ $25 \mathrm{~mm}$. Prior to bonding, all substrate surfaces were prepared by polishing down to 2400 grit emery paper, degreasing with acetone and electrochemically treated using Phosphoric Acid Anodizing (PAA: $20 \mathrm{~min}, 10 \% \mathrm{wt} . \mathrm{H}_{3} \mathrm{PO}_{4}, 20 \mathrm{~V}$ direct current, with a titanium grid and 15 $\mathrm{mm}$ electrode distance). In preparation, the upper surface of the thin adherend was mirror finished to permit precise profilometry measurements. The aluminium plates were bonded together using a commercial epoxy resin (Bostik, La Défense, Paris, France) consisting of bisphenol A of average molecular weight $<700$, cured with N(3 dimethylaminopropyl) $-1,3$ propylenediamine. This material had a Young's modulus ca. 3.0 GPa, and Poisson's ratio was assumed to be 0.37 , as stated in the supplier's data sheet. Crosslinking was effected at ambient temperature $\left(c a .23^{\circ} \mathrm{C}\right)$ for 24 hours under 0.3 bar pressure and at $c a .55 \% \mathrm{RH}$. Samples were left for a few days in usual ambient conditions before testing. Two bondline thicknesses were studied: $e=0.2 \pm 0.05 \mathrm{~mm}$ and $e=0.8 \pm 0.05 \mathrm{~mm}$. The values of thickness were fixed by inserting PTFE spacers at the joint extremities, prior to crosslinking. The bondline thickness was found to be homogeneous, as measured by optical microscopy. Various values of wedge thickness were employed to initiate crack propagation; for $e=0.2$ $\mathrm{mm}, 3 \mathrm{~mm}$ thick wedge was used. For $e=0.8 \mathrm{~mm}$, wedge thicknesses, $\Delta_{w}$, of $6 \mathrm{~mm}$ was used. Note that the separation distance: $\Delta=\Delta_{w}-e$, is used in the calculations and modelling, thus giving: $z(x=0)=\Delta$. 


\section{Profilometry}

After loading the adhesive joint by the wedge insertion and allowing time for any possible crack stabilisation, the deflection of the thin adherend was measured using a laser profilometer. An AltiSurf®500 surface characterization workstation (AltiMet, Thonon-LesBains, France) was adopted. The AltiSurf ${ }^{\circledR}$ system was mounted on a granite base. Samples were mounted to the measurement sliding table, made up of two $X$ and Y DC motorized tables. X and Y resolution used was $2 \mu \mathrm{m}$. X steps were chosen to be $10 \mu \mathrm{m}$. The extent of the accessible area - Scan zone, in the longitudinal and transverse directions, was limited by that of the (vertical) Z-axis. An AltiProbe ${ }^{\circledR}$ high-resolution white light sensor was used. This was fitted with an optical unit functioning on the chromatic aberration principle. The measurement range of this sensor was $350 \mu \mathrm{m}$ with a vertical resolution of $30 \mathrm{~nm}$. Two measurements were made for each sample point. The first was made on the unloaded sample and the second after loading. The experimental set-up is shown in Fig.1.

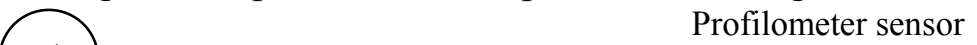

a)

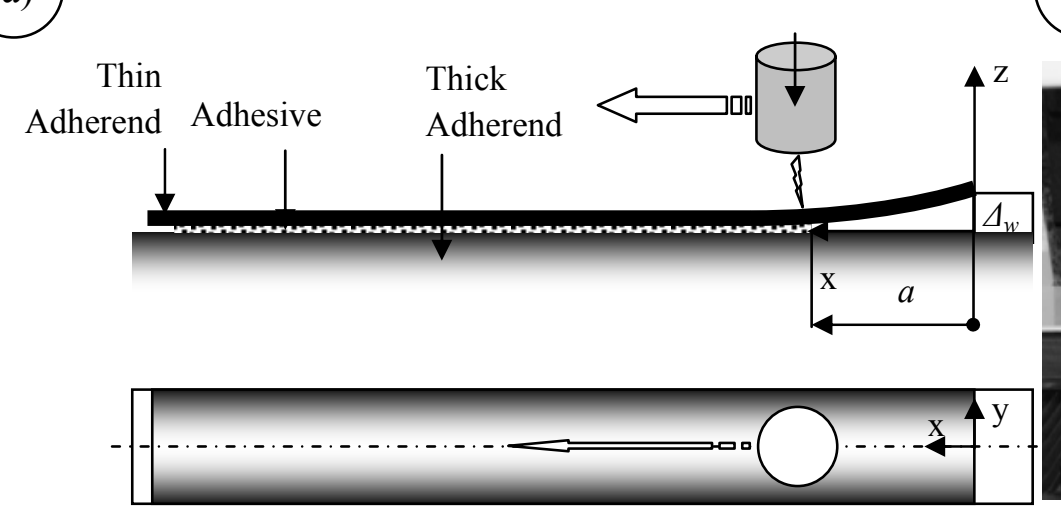

b)

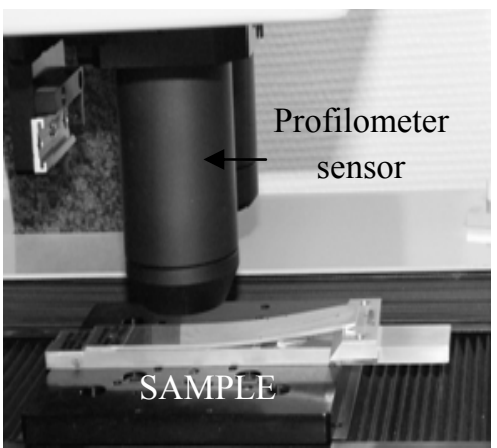

Fig. 1. (a) Principle of the profilometry measurements and (b) sample during test

\section{Euler-Bernoulli model (rigid foundation)}

The classic method for assessment of the adhesive fracture energy, in a wedge or related test, is based upon the cantilever beam and employs the Euler-Bernoulli equations. In our case, the system is asymmetric: the lower adherend is considered rigid. We introduce Cartesian coordinates, $(x, z)$, representing respectively distance from the wedge along the bondline and (vertical) displacement. Reduction of the bonded system, loaded after insertion of a wedge, to that of a cantilever beam is shown schematically in Fig.2. The brick wall shows schematically that the beam is considered encastré for $x>a$.
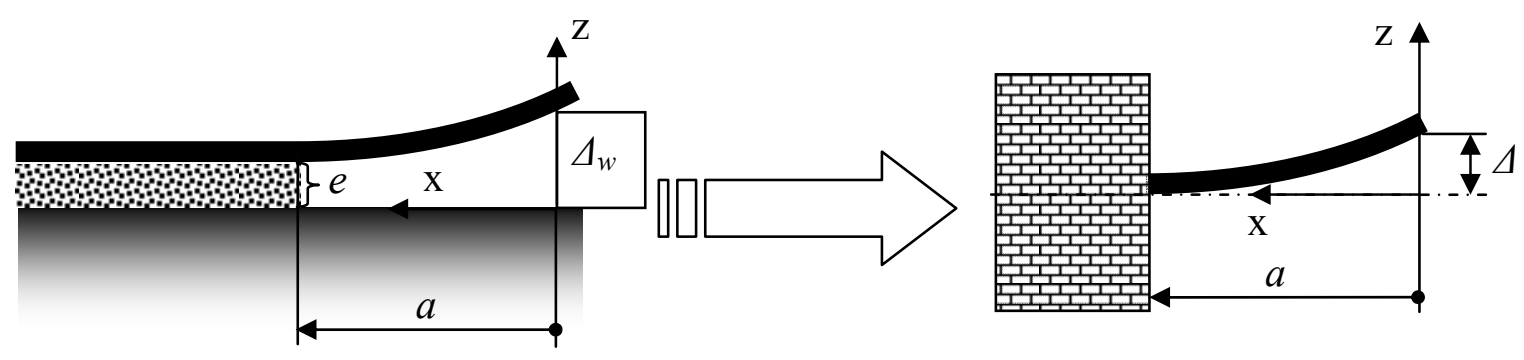

Fig. 2. Representation of the adhesive joint by Euler-Bernoulli model 
The bending moment, $M$, given by $F x$, is related to beam curvature, $d^{2} z / d x^{2}$, by:

$$
\frac{d^{2} z}{d x^{2}} E I=F x
$$

where $F$ is the only force acting perpendicularly to the $x$ axis, which has its origin at the wedge, as shown, and EI is the product of Young's modulus and second moment of the beam section. After integration and use of boundary conditions $z(a)=d z / d x(a)=0$, we obtain the displacement, or deflection, $z(x)$ :

$$
\left.z(x)\right|_{0} ^{a}=\frac{F}{E I}\left(\frac{1}{6} x^{3}-\frac{1}{2} a^{2} x+\frac{1}{3} a^{3}\right)
$$

We may express the deflection at the origin, $\Delta$, as follows:

$$
z(x=0)=\Delta=\frac{F a^{3}}{3 E I}
$$

to obtain:

$$
\left.z(x)\right|_{0} ^{a}=3 \Delta\left[\frac{1}{6}\left(\frac{x}{a}\right)^{3}-\frac{1}{2}\left(\frac{x}{a}\right)+\frac{1}{3}\right]
$$

The form of the beam deflection, given by equation (2) or (4), is simple. This is the main reason why this classical approach is so popular. The derivation represents a simple and efficient way of estimating the fracture properties of adhesive joints from a knowledge of $\Delta$ and $a$.

\section{Winkler model (one parameter elastic foundation)}

Winkler approach was apparently the first developed to describe the root rotation effect [14] and has recently been employed to consider the influence of adhesive compliance [15]. Considering again Fig.2., we take a beam bonded in the range $a<x<+\infty$, and free over $0<x$ $<a$. In terms of the Winkler model, the situation can be reduced to that shown schematically in Fig.3., where the bonded section has some flexibility, and therefore strains to some extent in the direction parallel to the applied force, along $z$. This elastic strain may be modelled by Hook spring elements, of stiffness $k$. (The applied load per unit length and at coordinate $x$, $q(x)$, is then given by $q(x)=-k z(x)$, where $z$ is the vertical displacement of the bonded element). 


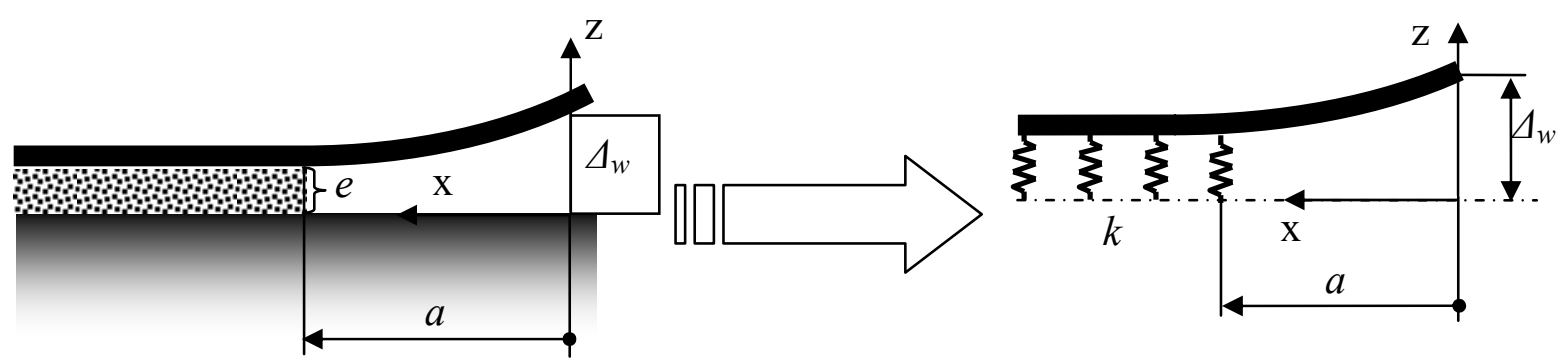

Fig. 3. Representation of the adhesive joint corresponding to Winkler model

The differential equation for the part of the beam laying on the elastic foundation is then:

$$
\frac{d^{4} z}{d x^{4}}+\frac{k}{E I} z=0 ; a<x<+\infty
$$

The general solution of this differential equation involves 4 arbitrary constants, but parts of the solution are physically unrealistic, when $k>0$, leading to divergence at infinity. Terms in $e^{\lambda(x-a)}$ are thus neglected, leading to:

$$
\left.z(x)\right|_{a} ^{+\infty}=e^{\lambda(a-x)}\left[A_{1} \cos \lambda(a-x)+B_{1} \sin \lambda(a-x)\right] ; \quad a<x<+\infty
$$

For the region $0<\mathrm{x}<a$, we have $k=0$, and therefore:

$$
\frac{d^{4} z}{d x^{4}}=0 ; 0<\mathrm{x}<a
$$

This has a straightforward polynomial solution:

$$
\left.z(x)\right|_{0} ^{a}=A_{2} x^{3}+B_{2} x^{2}+C_{2} x+D_{2}
$$

The solution for $a<x<+\infty$, equation (6), contains an intrinsic parameter, $\lambda\left(\mathrm{mm}^{-1}\right)$, which we term wave number, by analogy with infra red spectra, acoustics and fields involving propagation, and because of the units:

$$
\lambda=\frac{\sqrt{2}}{2}\left(\frac{k}{E I}\right)^{1 / 4}
$$

The term $k$ is often expressed as $E_{a d h} . b / e$, where $E_{a d h}$ is Young's modulus of the adhesive, $e$ its initial thickness and $b$ is joint width. Taking into account the influence of Poisson's ratio (confinement), we have [15]:

$$
k=\frac{1-v_{a d h}}{\left(1-2 v_{a d h}\right)\left(1+v_{a d h}\right)} \frac{b E_{a d h}}{e}
$$


The bracketed terms in $v_{\mathrm{A}}$ are to allow for essentially plane strain conditions existing in a thin adhesive layer $(e / b<<1)$. The correction is not negligible, since an adhesive could easily have a Poisson's ratio of 0.4 , leading to $c a$. twice the value of $k$ given by the simpler, often used, expression. Constants $\mathrm{A}_{1}, \mathrm{~B}_{1}, \mathrm{~A}_{2}, \mathrm{~B}_{2}, \mathrm{C}_{2}$, and $\mathrm{D}_{2}$ can be found from boundary conditions of geometry and continuity of functions (6) and (8) and their derivatives up to order 3 . This leads to:

$$
\begin{aligned}
& A_{1}=\Delta \frac{3(1+\lambda a)}{\left(3+6 \lambda a+6 \lambda^{2} a^{2}+2 \lambda^{3} a^{3}\right)} \\
& B_{1}=\Delta \frac{3 \lambda a}{\left(3+6 \lambda a+6 \lambda^{2} a^{2}+2 \lambda^{3} a^{3}\right)} \\
& A_{2}=\Delta \frac{\lambda^{3}}{\left(3+6 \lambda a+6 \lambda^{2} a^{2}+2 \lambda^{3} a^{3}\right)} \\
& B_{2}=0 \\
& C_{2}=\Delta \frac{-3 \lambda(1+a \lambda)^{2}}{\left(3+6 \lambda a+6 \lambda^{2} a^{2}+2 \lambda^{3} a^{3}\right)} \\
& D_{2}=\Delta
\end{aligned}
$$

\section{Finite element analysis}

Further contribution to the elastic foundation model of the asymmetric wedge test has been undertaken using the CAST3M finite element programme to simulate the experiment and calculate the deflection of the beam on an elastic layer. To do this, the beam is discretised with SEG2 - Timoshenko beam elements, to prevent for any blockage at the junction between the adherend and free zone (a classical numerical artefact in the finite element beam model). The adhesive is represented with QUA4 linear elements, which are recommended for high stress singularities. The simplified situation and reduction of the bonded system to the FEA model proposed is shown schematically in Fig.4a.

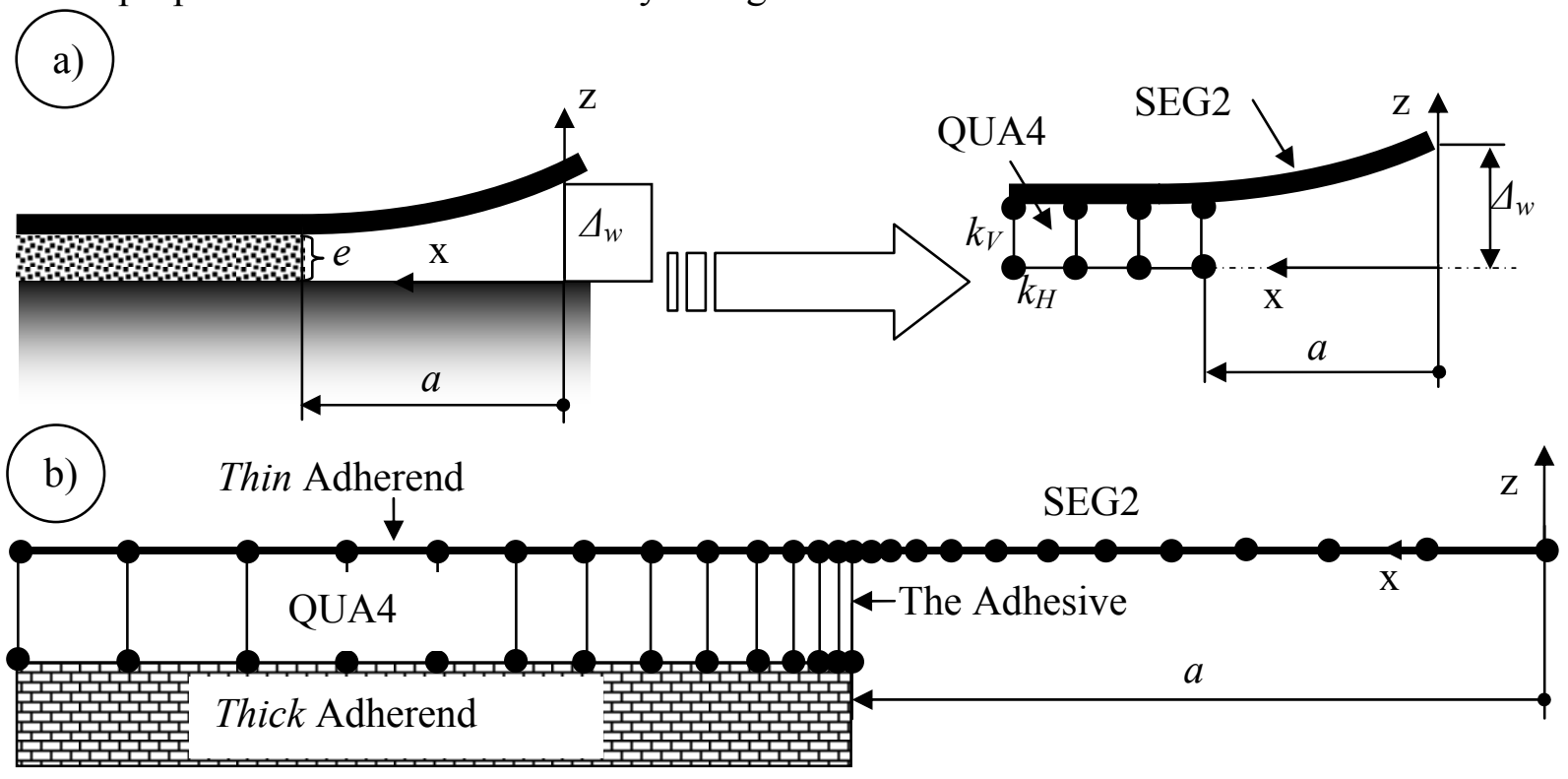




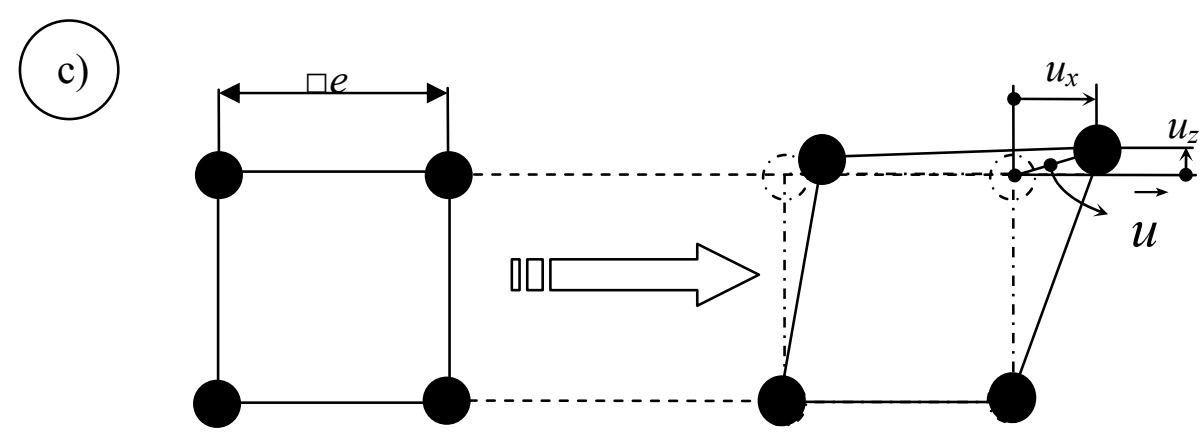

Fig. 4. 2D FEM of the adhesive joint. (a) Reduction of the adhesive joint to 2D Finite Element model. (b) Details of the FEA model used. (c) 2D deformation of the finite element after applying opening load

The adhesive elements, of initial thickness between the adherends, $e$, are assumed perfectly clamped at the bottom face (brick wall representation in Fig.4b.). QUA4 linear elements provide the simplest model for a thin layer behaving linearly elastically, with tensile $\left(k_{V}\right)$ and shear $\left(k_{H}\right)$ rigidity. The plate-adhesive interface was modelled using the COLLER procedure (operator), which relates the displacement of the upper surface of the adhesive (interface with upper adherend) to the lower surface of the adherend (rather than the midline of the beam). This function allows us to bond two surfaces, leaving the possibility for edge rotations and displacements, without separation of the bonded elements. An additional parameter could be taken into account, viz. the eccentricity of the flexible adherend with respect to the adhesive layer. The bent beam is represented in FEA by the position of its mean plane. In practice, it is the bottom side of the plate that is in contact with the adherend, so that the displacement imposed on the adhesive would be more correctly given by:

$$
\vec{u}=\left(u_{x}+\frac{h}{2} \theta_{z}\right) \vec{x}+u_{x} \vec{y}
$$

where $u_{x}$ is the local beam displacement along $x$ and $\theta_{z}$ is the local beam rotation. The mesh was constructed of 50 finite elements (FE), element length increasing with distance, $|x|$, from the crack front in both directions. The first element was of length $s_{l}=100 \mu \mathrm{m}$. The FEA model is shown in Fig.4b. and a schematic representation of finite element deformation in Fig.4c.

\section{RESULTS AND DISCUSSION}

\section{Crack length estimation from profilometry measurements}

A very sensitive profilometry sensor was used (N.B. the $z$-range was $350 \mu \mathrm{m}$ ). As a consequence, deflection of the entire plate could not be not measured (simultaneously). Deflection measurements were therefore restricted to the immediate neighbourhood of the zone within which the crack front was expected to be, so called scan zone (Fig.5.). At this stage, the $x$ axis refers uniquely to the range of scan, and not wedge position. The profilogramme shown includes displacements from both free and bonded parts of the joint, which are separated by a bold line. 


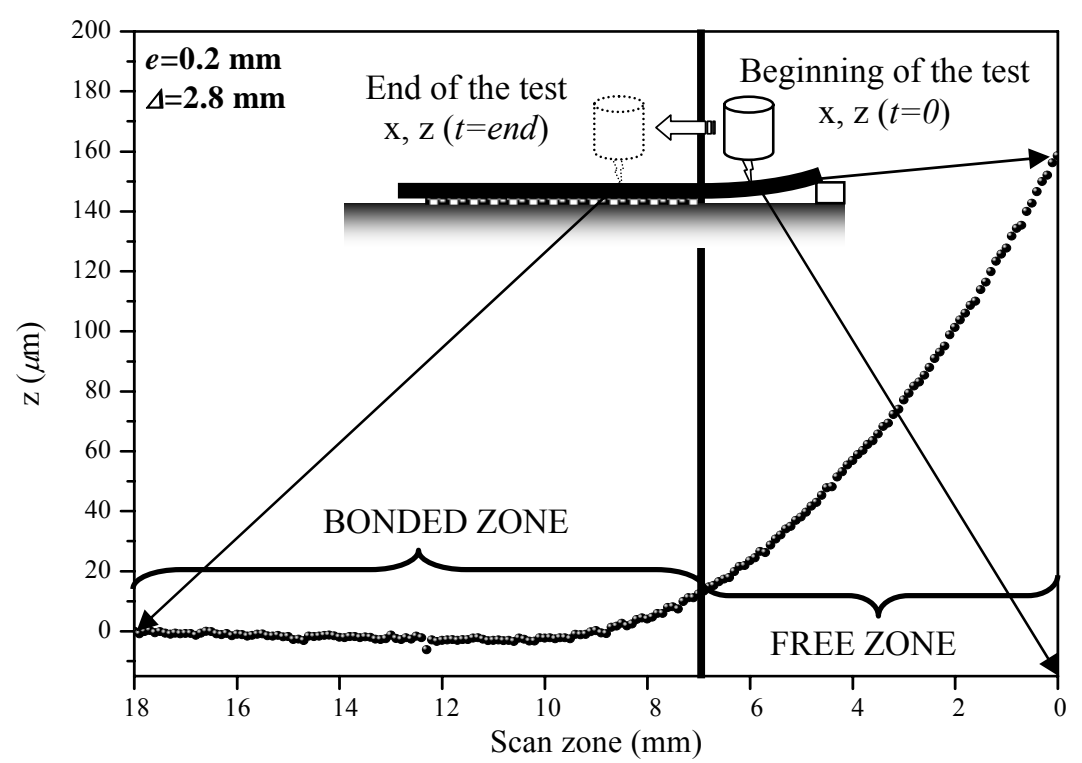

Fig. 5. Profilogramme of the zone in the vicinity of crack front

These measurements correspond to the centre line of the flexible adherend (in the plane of symmetry). Note the slight negative deflection, or compression of the adhesive layer, some way into the bonded zone, due to the relative rigidity of the thin adherend resisting the bending moment. Further on, there is recovery (less compression). There is a wave component to the deflection and this is the qualitative, physical explanation of the presence of trigonometric functions in equation (6). The free zone part of the graph was used to estimate the actual crack position. This was done using a $3^{\text {rd }}$ order polynomial fit, in keeping with both the basic Euler-Bernoulli and Winkler model equations for this part of the joint - equation (8). The results of the fitting are represented on Fig.6. for the cases studied.
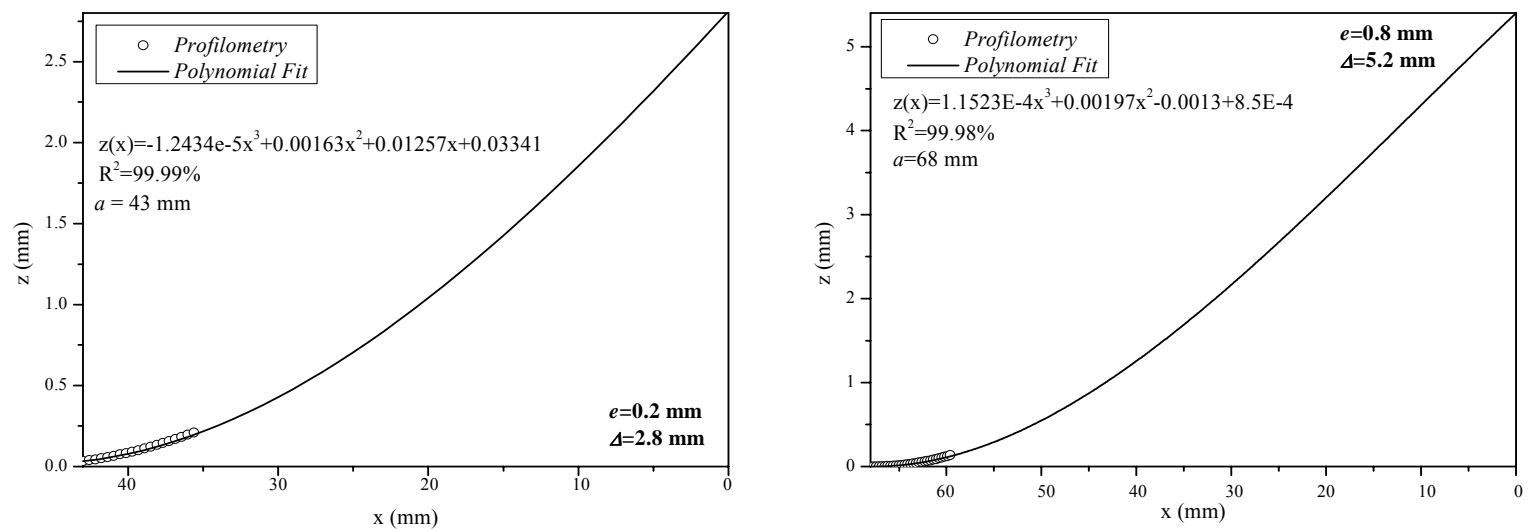

Fig. 6. Polynomial fit of crack length. (Left) For the thin adhesive layer, $a=43 \mathrm{~mm}$. (Right) For thicker bondline $a=68 \mathrm{~mm}$

Polynomial functions thus obtained were extrapolated to give the $z$ deflection corresponding to the wedge thickness, thus allowing the approximate crack length to be deduced. This operation leads to $43 \mathrm{~mm}$ and $68 \mathrm{~mm}$ of the crack length for the thinner and thicker of bondline respectively.

\section{Comparison of models with profilometry}

In Fig.7., we present the $z$ deflection $v s x$ for the bonded zone for various cases studied (note the different scales). No results are shown for the Euler-Bernoulli model within the bonded 
zone, since the hypothesis is of an encastré beam, and therefore we would simply have $z=0$. However, as clearly shown by the profilometry results, and in qualitative agreement with the Winkler theory, there is a distinct dip in the profile near the bond edge, restored further into the joint. There are, however, quantitative differences between the experimental deflections and those expected from Winkler theory, especially for the thicker bondline, when the adhesive is playing a more important role. As can be seen, the agreement for both models is good outside the bonded area, although the Winkler model generally overestimates slightly, showing higher deflections than given by experiment. In the bonded zone the Winkler model gives an approximate account of what occurs: it is clearly a great improvement on the encastré analysis. In both cases FEM results gives better approximation of the experimental results. However, at this point, we can assume that Winkler model could be treated as sufficient enough to predict joint behaviour.
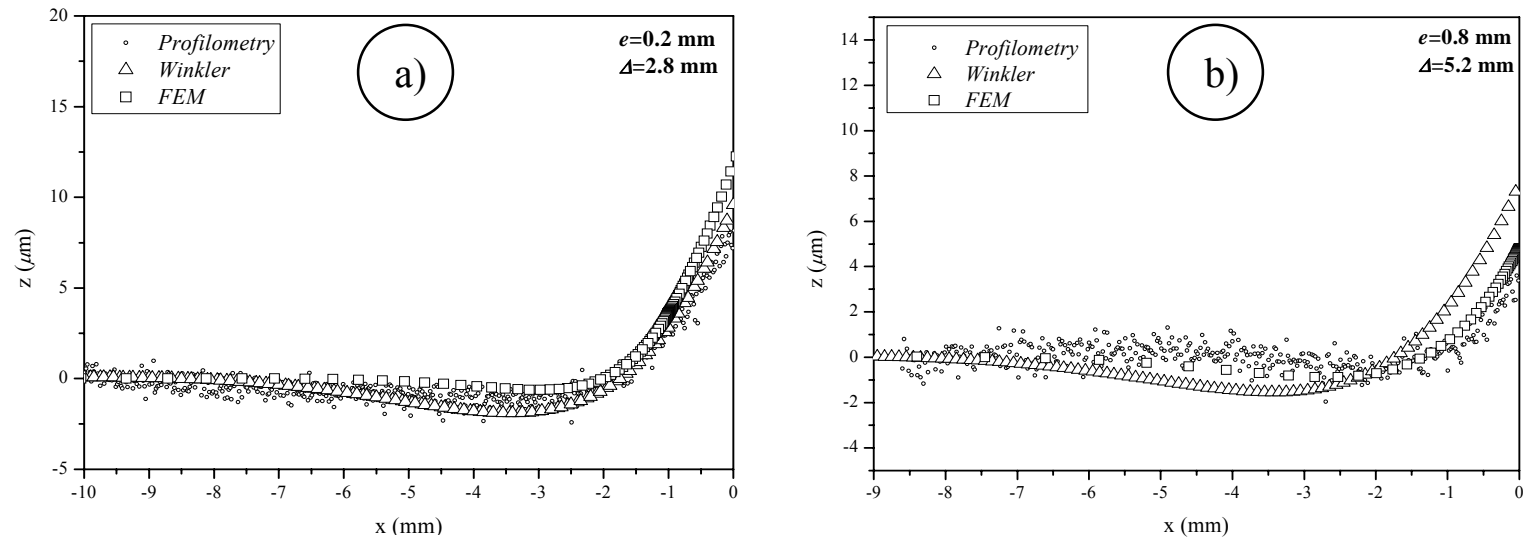

Fig. 7. Bonded zones deflections for two cases studied, a) thin bondline, b) thick bondline

\section{Fracture Energy}

For most purposes, the most important parameter estimated from fracture tests is the energy release rate, which, while the crack is propagating, equates with fracture energy. In general, the elastic adhesive fracture energy can be found from the classical energy balance expression:

$$
G=-\frac{1}{b} \frac{\partial U}{\partial a}
$$

where $U$ is the elastic energy stored and $b$ is beam width.

In the present context, we may express the strain energy release rate in the case of the classic Euler-Bernoulli beam model (EB) as:

$$
G_{E B}=\frac{3}{8} \frac{E h^{3} \Delta^{2}}{a^{4}}
$$

For the Winkler (W) solution, we have [15]: 


$$
G_{W}=\frac{3}{2} E h^{3} \Delta^{2} \lambda^{4} \frac{1+2 \lambda a+\lambda^{2} a^{2}}{\left(3+6 \lambda a+6 \lambda^{2} a^{2}+2 \lambda^{3} a^{3}\right)^{2}}
$$

For the finite element solution (FEA), we use the classic energy balance equation (13). The energy release rate calculated is the change in the elastic energy (stored within the flexible beam $(U)$ ) while the crack propagates by $\delta a$, from position $a_{i}$ to the position $a_{i+1}$, thus:

$$
G_{F E A}=-\frac{1}{b} \frac{\partial U}{\partial a}=-\frac{1}{b} \frac{U\left(a_{i+1}\right)-U\left(a_{i}\right)}{a_{i+1}-a_{i}}=-\frac{1}{b} \frac{\delta U}{\delta a}
$$

In Table 1, the energy release rate values are presented for the situations discussed above, and for values of the various parameters studied experimentally. Assuming that the FEA model gives the best approximation to the real energy release rate, in our case at least, the standard Euler-Bernoulli analysis overestimates energy release rate (and therefore fracture energy in the case of a propagating crack). This is particularly noticeable in the first example given in Table 1 , in which the overestimation is nearly $16 \%$ (note that this case corresponds to the smaller of the crack lengths).

Table 1. Results of the analysis performed

\begin{tabular}{|c|c|c|c|}
\hline Input data & $\begin{array}{c}\text { Euler-Bernoulli } \\
G_{E B}\left(\mathrm{Jm}^{-2}\right)\end{array}$ & $\begin{array}{c}\text { Winkler } \\
G_{W}\left(\mathrm{Jm}^{-2}\right)\end{array}$ & $\begin{array}{c}\text { 2D FEA } \\
G_{F E A}\left(\mathrm{Jm}^{-2}\right)\end{array}$ \\
\hline $\begin{array}{c}e=0.2 \mathrm{~mm} \\
\Delta=3 \mathrm{~mm} \\
a=43 \mathrm{~mm}\end{array}$ & 197.4 & 162.3 & 172.4 \\
\hline $\begin{array}{c}e=0.8 \mathrm{~mm} \\
\Delta=6 \mathrm{~mm} \\
a=68 \mathrm{~mm}\end{array}$ & 117.4 & 103.6 & 105.5 \\
\hline
\end{tabular}

The Winkler model tends to underestimate energy release rate, but at most by less than $10 \%$. Again taking the FEA model as the standard, we can show graphically the approximate error introduced by the models used. This representation for the experimental data is given in Fig.8. in the form of absolute errors, following the equations: $\left(G_{\text {model }}-G_{F E A} / G_{F E A}\right) \cdot 100 \%$.

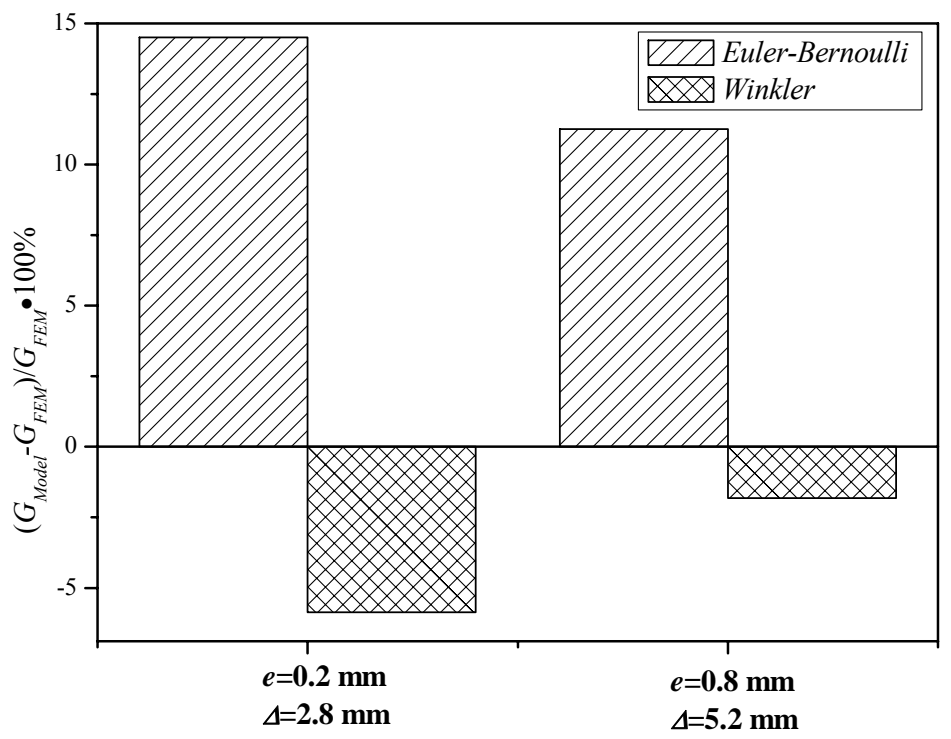

Fig. 8. Absolute error of the analytical solutions on fracture energy of asymmetric joint 
Finally, for the most common situations $(\lambda a<100)$, we can draw correction curves for the Euler-Bernoulli and Winkler models. These are shown in Fig.9. Naturally, when EulerBernoulli model is used the wave number, $\lambda$ vanish.

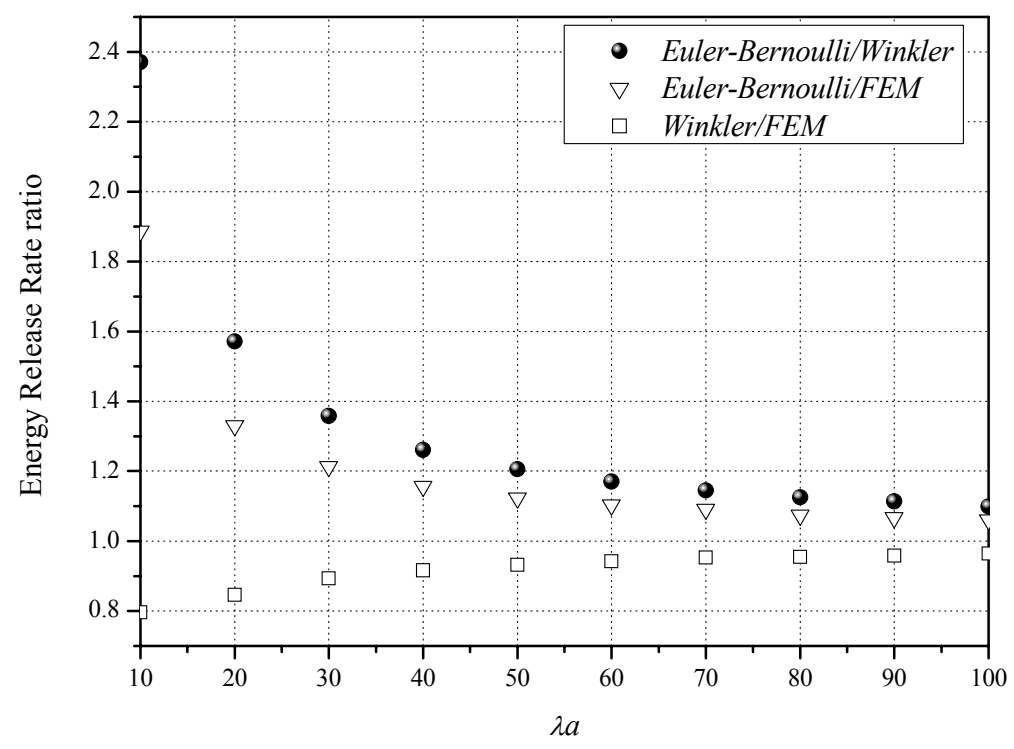

Fig. 9. Correction factors curves for the models studied

\section{CONCLUSIONS}

New experimental technique was introduced, laser profilometry, to study joint behaviour and shearing effect in asymmetric bonded joints. This technique allows detailed studies of the bonded zone in the vicinity of the crack front when joint is submitted to mode I loading. Finite Element Model was built and compared with two commonly used analytical solutions: Euler-Bernoulli and Winkler. It was concluded that complex: shearing - cleavage compliance effects the adhesive and fracture properties of the joint. Analytical models used nowadays requires introduction of appropriate correction factors. These, following power law fitting (using Matlab®2008a, Curve Fitting Toolbox) were found:

a) for Euler-Bernoulli to Winkler correction the factor is:

$$
\frac{G_{E-B}}{G_{\text {Winkler }}}=26.72 \cdot \lambda a^{-1.302}+1.038
$$

b) for Euler-Bernoulli to Finite Element Analysis results the factor is:

$$
\frac{G_{E-B}}{G_{F E M}}=25.44 \cdot \lambda a^{-1.479}+1.04
$$


c) for Winkler to Finite Element Analysis results the factor is:

$$
\frac{G_{\text {Winkler }}}{G_{F E M}}=-0.7065 \cdot \lambda a^{-0.3116}+1.137
$$

All factors were achieved with coefficient of determination, $\mathrm{R}^{2}$ higher than $99 \%$ and provide easy way for raw fracture test data manipulation. Moreover, they allow efficient joint design with the minimal introduced errors. The new introduced here corrections emphasize another effect: the adhesive layer impact on the joint properties. The correction is function of two parameters: $a$ and $\lambda$. While change of the crack length with time: $a=f(t)$ is usual situation, correction could be used in case of second possible situation, namely change of the adhesive properties, viz. $\lambda=f(t)$. In particular situation this allow e.g. any adhesive degradation to be taken into account.

\section{ACKNOWLAGEMENTS}

Michal K. Budzik would like to thank the French National Centre for Scientific Research (CNRS) for the financial support within the CNRS grant.

\section{REFERENCES}

1. Allen K. W.: Papyrus - some ancient problems in bonding, International Journal of Adhesion and Adhesives, 16, pp. 47-51, 1996.

2. Licari J. J., Swanson D. W.: Adhesive Technology for electronic applications, William Andrew Publishing, 2005.

3. Täljsten B.: The Importance of Adhesive Bonding - An historic overview and future possibilities, Proceedings of the International Symposium on Bond Behaviour of FRP in Structures, ed. Chen and Teng, International Institute for FRP in Construction, 2005.

4. Higgins A.: Adhesive bonding of aircraft structures, International Journal of Adhesion and Adhesives, 20, pp. 367-376, 2000.

5. Erdman D., Battiste R., Boeman R., Klett L.: Characterization of a Structural Adhesive in Automotive Environments, Future Car Congress 2000, Engineering Technology Division, Oak Ridge National Laboratory, Society of Automotive Engineers, Inc., 2000.

6. Jie K., Rongchang N., Yusheng T.: Study on modification of epoxy resins with acrylate liquid rubber containing pendant epoxy groups, Journal of Material Science Letters, 41, pp. 1639-1641, 2006.

7. Rider A. N., Olsson-Jacques C. L., Arnott D. R.: Influence of Adherend Surface Preparation on Bond Durability, Surface and Interface Analysis, 27, pp. 1055-1063, 1999. 
8. Budzik M. K., Jumel J., Imielinska K., Shanahan M. E. R.: Accurate and continuous adhesive fracture energy determination using an instrumented wedge test, International Journal of Adhesion and Adhesives 29, pp. 694-701, 2009.

9. Chen C. R., Kolednik O., Scheider I., Siegmund T., Tatschl A., Fischer F. D.: On the determination of the cohesive zone parameters for the modeling of micro-ductile crack growth in thick specimens, International Journal of Fracture, 120, pp. 517-536, 2003.

10. Fernlund G.: Stress analysis of bonded lap joints using fracture mechanics and energy balance, International Journal of Adhesion and Adhesives, 27, pp. 584-592, 2007.

11. Ikegami K., Fujii T., Kawagoe H., Kyogoku H., Motoie K., Nohno K., Sugibayashi T., Yoshida F.: Benchmark tests on adhesive strengths in butt, single and double lap joints and double-cantilever beams, International Journal of Adhesion and Adhesives, 16, pp. 219-226, 1996.

12. Tschegg E. K., Krassnitzer T.: Mode I fracturing properties of epoxy bonding paste, International Journal of Adhesion and Adhesives, 28, pp. 340-349, 2008.

13. Blackman B. R. K., Kinloch A. J., Paraschi M., Teo W.S.: Measuring the mode I adhesive fracture energy, $G_{I C}$, of structural adhesive joints: the results of an international roundrobin, International Journal of Adhesion and Adhesives, 23, pp. 293-305, 2003.

14. Cotterell B., Hbaieb K., Williams J. G., Hadavinia H., Tropsa V.: The root rotation in double cantilever beam and peel tests, Mechanics of Materials, 38, pp. 571-584, 2006.

15. Budzik M. K., Jumel J., Imielinska K., Shanahan M. E. R.: Effect of Adhesive Compliance in the Assessment of Soft Adhesives with the Wedge Test, Journal of Adhesion Science and Technology 0, pp. 1-19, 2010.

16. Jian S., Jun S., Zengjie D., Huijue Z.: The finite element analysis of deformation and stress triaxiality of a mixed I + II mode with elasticplastic crack tip, International Journal of Fracture, 87, pp. 47-58, 1997.

17. Chen B., Dillard D. A.: The effect of the $T$-stress on crack path selection in adhesively bonded joints, International Journal of Adhesion and Adhesives, 21, pp. 357-368, 2001. 